\title{
GMR
}

\section{Phylogenetic analysis of DNA and RNA polymerases from a Moniliophthora perniciosa mitochondrial plasmid reveals probable lateral gene transfer}

\author{
B.S. Andrade ${ }^{1}$ and A. Góes-Neto ${ }^{2}$ \\ 1'Departamento de Ciências Biológicas, \\ Universidade Estadual do Sudoeste da Bahia, Jequié, BA, Brasil \\ 'Laboratório de Pesquisa em Microbiologia, Departamento de Ciências Biológicas, \\ Universidade Estadual de Feira de Santana, Feira de Santana, BA, Brasil \\ Corresponding author: B.S. Andrade \\ E-mail: bandrade@uesb.edu.br
}

Genet. Mol. Res. 14 (4): 14105-14114 (2015)

Received May 29, 2015

Accepted August 13, 2015

Published October 29, 2015

DOI http://dx.doi.org/10.4238/2015.October.29.30

ABSTRACT. The filamentous fungus Moniliophthora perniciosa is a hemibiotrophic basidiomycete that causes witches' broom disease of cacao (Theobroma cacao L.). Many fungal mitochondrial plasmids are DNA and RNA polymerase-encoding invertrons with terminal inverted repeats and 5'-linked proteins. The aim of this study was to carry out comparative and phylogenetic analyses of DNA and RNA polymerases for all known linear mitochondrial plasmids in fungi. We performed these analyses at both gene and protein levels and assessed differences between fungal and viral polymerases in order to test the lateral gene transfer (LGT) hypothesis. We analyzed all mitochondrial plasmids of the invertron type within the fungal clade, including five from Ascomycota, seven from Basidiomycota, and one from Chytridiomycota. All phylogenetic analyses generated similar tree topologies regardless of the methods and datasets used. It is likely that DNA and RNA polymerase genes were inserted into the mitochondrial genomes 
of the 13 fungal species examined in our study as a result of different LGT events. These findings are important for a better understanding of the evolutionary relationships between fungal mitochondrial plasmids.

Key words: Moniliophthora perniciosa; Mitochondrial plasmids; Fungi; Molecular phylogeny

\section{INTRODUCTION}

The basidiomycete Moniliophthora perniciosa (Stahel) Aime \& Phillips-Mora, previously known as Crinipellis perniciosa (Stahel) Singer, is the causative agent of witches' broom disease of the cacao tree (Theobroma cacao L.), whose seeds are used for the production of chocolate (Aime and Phillips-Mora, 2005). It is the most important phytopathological problem affecting cacao-producing areas in the American continent, and has decimated the Brazilian cacao industry (Griffith et al., 2003). M. perniciosa is classified in the order Agaricales, a group including few known pathogens (Aime and Phillips-Mora, 2005), and is endemic in the Amazon region. It is the only pathogen that develops concurrently with the cocoa plant (Purdy and Schmidt, 1996). Several biotypes of $M$. perniciosa have been described, with biotype $C$ affecting $T$. cacao and other species of the genera Theobroma and Herrania (Griffith and Hedger, 1994). The mitochondrial (Formighieri et al., 2008) and whole genomes (Mondego et al., 2008) of this fungus have been sequenced.

Plasmids are short lengths of DNA or RNA with the ability to replicate inside living cells, independently of the host genome (Cahan and Kennel, 2005). These structures are also able to covalently integrate into cellular and organellar genomes and thus be replicated along with host DNA (Griffiths, 1995). Plasmids were originally discovered in bacteria but similar molecules have subsequently been found in eukaryotes, predominantly in the mitochondria of plants and filamentous fungi (Cahan and Kennel, 2005). These molecules exist in circular or linear conformations (Griffiths, 1995), and most eukaryotic plasmids are of the latter form (Meinhardt et al., 1990). Mitochondrial plasmids are generally viewed as being intracellular parasites, and their prevalence, at least in filamentous fungal hosts, corresponds to their ability to spread horizontally via hyphal anastomosis (Cahan and Kennel, 2005). In most cases, the impact on the host phenotype of plasmid presence or introgression is not clear. However, these molecules may confer some selective advantage for the host, modifying aspects of metabolism or mitochondrial division. In many physiological studies, no changes in the rate or pattern of growth have been observed because no tests sufficiently sensitive to identify very small changes in growth, respiration, or reproductive rate exist (Griffiths, 1995). However, a phenomenon known as fungal senescence syndrome has been described in some species of the genus Neurospora (Marcou, 1961; Chan et al., 1991), involving progressive loss of mitochondrial function, loss of conidiogenesis, and conidial viability, together with reduction of hyphal growth and potentially, death of the mycelium (Meinhardt et al., 1990; Chan et al., 1991). Mitochondrial plasmids are widely distributed in filamentous fungi and exhibit some common features, such as the presence of terminal inverted repeats (TIRs) and genes encoding DNA and RNA polymerases (DPO and RPO, respectively; Kempken et al., 1992; Kempken, 1994; Cahan and Kennel, 2005). Such plasmids are found in various Ascomycota and Basidiomycota species, including several saprophytes and plant pathogens (Giese et al., 2003), particularly in the genus Neurospora (Xu et al., 1999). They are often transmitted in the same manner as mitochondria and mitochondrial DNA, and during sexual reproduction, maternal plasmids are inherited by most or all of the resulting progeny. According to the endosymbiosis theory, plasmids in fungal mitochondria 
are of prokaryotic origin, and some genes may have been inherited from bacteriophages (Griffiths, 1995). Relationships between mitochondrial plasmids from different species have been estimated using DPO and RPO nucleotide and amino acid sequences. The transmission of mitochondrial plasmids during reproduction in fungi has been the subject of many studies (Giese et al., 2003), including investigations of introgression in Neurospora (Bok et al., 1999) and asexual transmission in Cryphonectria parasitica (Murrill) ME Barr (Baidyaroy et al., 2000). Other research has shown that despite the existence of somatic incompatibility barriers, plasmid transmission has also occurred by anastomosis (Giese et al., 2003). The M. perniciosa mitochondrial plasmid has a typical invertron structure and contains DPO- and RPO-encoding genes in opposite orientations. Furthermore, it appears to be stably integrated into the mitochondrial genome and is probably involved in senescence (Formighieri et al., 2008).

In the present study, we analyzed the DNA and protein sequences of DNA and RNA polymerase enzymes encoded by all known fungal mitochondrial linear plasmids, including those from 13 species: seven ascomycetes, five basidiomycetes (of which, M. perniciosa) and one chytridiomycotan. Our aim was to generate a phylogeny based on molecular data to test the hypothesis that lateral transfer of polymerase genes has occurred within the fungal clade and between viruses and fungi.

\section{MATERIAL AND METHODS}

Phylogenetic analyses were performed using DPO and RPO nucleotide and amino acid sequences for all fungal mitochondrial linear plasmids available in the NCBI/EMBL/DDBJ databases. In addition, protein sequences of viral DPOs and RPOs were included in a comparative similarity analysis using BLAST version 2.2.16 (Altschul et al., 1997), owing to their probable evolutionary relationship with polymerases encoded by fungal mitochondrial plasmids.

DPO and RPO nucleotide sequences for the 13 fungal plasmids were codon-aligned with their corresponding amino acid sequences using PAL2NAL (Suyama et al., 2006). The resulting alignments were edited in BioEdit 7.0.5.2 (Hall, 1999) and saved in NEXUS format as DPO/RPO contigs. After these nucleotide contigs had been assessed using Modeltest (Posada and Crandall, 1998), general time-reversible + gamma + invariant sites was selected as the most appropriate evolutionary model for the maximum likelihood analysis matrix. Clade robustness was measured using bootstrap proportions (1000 pseudoreplicates) and phylograms were generated in PAUP 4.0b10 (Swofford, 2002) before being saved and visualized in TreeView 1.6.6 (Page, 1996). For these analyses, the outgroup was represented by DPO and RPO sequences from the Spizellomyces punctatus (WJ Koch) DJS Barr mitochondrial plasmid, since chytridiomycotan species likely originated before their Ascomycota and Basidiomycota counterparts (James et al., 2006).

The amino acid sequences of fungal and viral DPOs and RPOs were aligned separately in BioEdit 7.0.5.2 using a BLOSUM62 matrix (Henikoff and Henikoff, 1992) and bootstrap proportions from 1000 pseudoreplicates. It was not possible to perform a combined analysis of DPO/RPO contigs using fungal and viral sequences, as these polymerases are not found together within the same viral species. A phylogeny of fungal and viral DPO and RPO amino acid sequences was generated by Bayesian analysis performed in MrBayes 3.1.2 (Ronquist and Huelsenbeck, 2003) using the evolutionary model Mtmam (Yang et al., 1998). This model was chosen as both fungal and mammalian mitochondrial genomes demonstrate equivalent substitution rates and similarities in codon usage compared to the standard nuclear genome (Osawa et al., 1992). Three 
independent runs were conducted, each with four chains, for $1 \times 10^{6}$ generations, sampling every 100 generations. In phylogenies of viral and fungal nucleotide sequences, bacteriophage phi29 DPO and T7 RPO were used as outgroups for DNA and RNA polymerase analyses, respectively.

\section{RESULTS}

Thirteen completely sequenced fungal mitochondrial plasmids exhibit a linear structure and TIRs: five in Ascomycota, seven in Basidiomycota and one in Chytridiomycota (Table 1). Plasmid size ranged from 1.7 to $8.6 \mathrm{~kb}$, with that of $S$. punctatus being the smallest and that of $N$. intermedia the largest. All fungal plasmids studied included DPO and RPO genes on opposite strands, except for that of $S$. punctatus, which encodes these proteins on the same strand. Comparative analyses of DPO and RPO proteins revealed a high degree of similarity between viral (Table 2) and fungal sequences. Maximum likelihood phylogenetic analysis of DPO/RPO nucleotide contigs generated a phylogram in which Ascomycota sequences were clearly grouped amongst those of Basidiomycota (Figure 1).

Table 1. Completely sequenced invertron-like linear mitochondrial plasmids in fungi.

\begin{tabular}{lllccccl}
\hline Plasmid & Fungus & Phylum & Size (bp) & DNA pol. (aa) & RNA pol. (aa) & Accession No. & Reference \\
\hline pEM & Agaricus bitorquis & Basidiomycota & 5810 & 797 & 1102 & X63075 & Robison and Horgen, 1999 \\
pBgh & Blumeria graminis f. sp hordei & Ascomycota & 7965 & 1062 & 973 & NC_004935 & Giese et al., 2003 \\
pCIK1 & Claviceps purpurea & Ascomycota & 6752 & 1063 & 963 & X15648 & Oeser and Tudzynski, 1989 \\
pMp & Moniliophthora perniciosa & Basidiomycota & 6743 & 899 & 1028 & NC_005927 & Formighieri et al.,, 2008 \\
pFV1 & Flammulina velutipes & Basidiomycota & 7363 & 925 & 1168 & AB028633 & Nakai et al., 2000 \\
pG114 & Gelasinospora sp & Ascomycota & 8231 & 987 & 831 & L40494 & Yuewang et al., 1996 \\
pPK2 & Pichia kluyveri & Ascomycota & 7174 & 1118 & 992 & Y11606 & Blaisonneau et al., 1999 \\
pHC2 & Hebeloma circinans & Basidiomycota & 3229 & 858 & 209 & Y11504 & Bai et al., 1997 \\
pHarbin-3 & Neurospora crassa & Ascomycota & 7050 & 1021 & 896 & AF133505 & Xu et al., 1999 \\
pKalilo & Neurospora intermedia & Ascomycota & 8642 & 969 & 811 & X52106 & Chan et al.,1991 \\
pMLP2 & Pleurotus ostreatus & Basidiomycota & 7005 & 900 & 993 & AF355103 & Kim et al., 2000 \\
pAL2-1 & Podospora pauciseta & Ascomycota & 8395 & 1197 & 948 & X60707 & Hermanns and Osiewacz, 1992 \\
pSp & Spizellomyces punctatus & Chytridiomycota & 1775 & 361 & 218 & AF404303 & Forget et al., 2002 \\
\hline
\end{tabular}

pol. = polymerase; aa $=$ amino acids.

Table 2. Viral DNA and RNA polymerases used in the phylogenetic analyses.

\begin{tabular}{llcccll}
\hline Virus & Virus type & Pol. type & Size $(\mathrm{bp})$ & Size (aa) & Accession No. & Reference \\
\hline Bacteriophage Bam35c & Adenovirus & DPO & 2205 & 735 & NP_943751.1 & Ravantti et al., 2003 \\
Phage GIL16c & Adenovirus & DPO & 2259 & 753 & YP_224103.1 & Verheust et al., 2005 \\
Human adenovirus 12 & Adenovirus & DPO & 3567 & 1189 & AP_000112.1 & Davison et al., 2003 \\
Bacteriophage phi29 & Adenovirus & DPO & 1725 & 575 & 1XHX_A & Kamtekar et al., 2004 \\
Simian adenovirus A & Adenovírus & DPO & 3507 & 1169 & YP_067908.1 & Kovacs et al., 2004 \\
Bacteriophage phiYeO3-12 & Retrovirus & RPO & 2652 & 884 & NP_052071.1 & Pajunen et al., 2001 \\
Bacteriophage T3 & Retrovirus & RPO & 2652 & 884 & NP_523301.1 & Pajunen et al., 2002 \\
Phage K1F & Retrovirus & RPO & 2679 & 893 & YP_338094.1 & Scholl and Merril, 2005 \\
Phage gh-1 & Retrovirus & RPO & 2655 & 885 & NP_813747.1 & Kovalyova and Kropinski, 2003 \\
BPK11 & Retrovirus & RPO & 2718 & 906 & P18147 & Dietz et al., 1990 \\
Vibriophage VP4 & Retrovirus & RPO & 2649 & 883 & YP_249577.1 & Wang et al., 2005 \\
Phage Berlin & Retrovirus & RPO & 2649 & 883 & YP_918986.1 & Noelting et al., 2006 \\
Phage T7 & Retrovirus & RPO & 2649 & 833 & 1CEZ & Dunn and Studier, 1981 \\
\hline
\end{tabular}

pol. $=$ polymerase $;$ aa $=$ amino acids $;$ DPO $=$ DNA polymerase $; \mathrm{RPO}=$ RNA polymerase . 


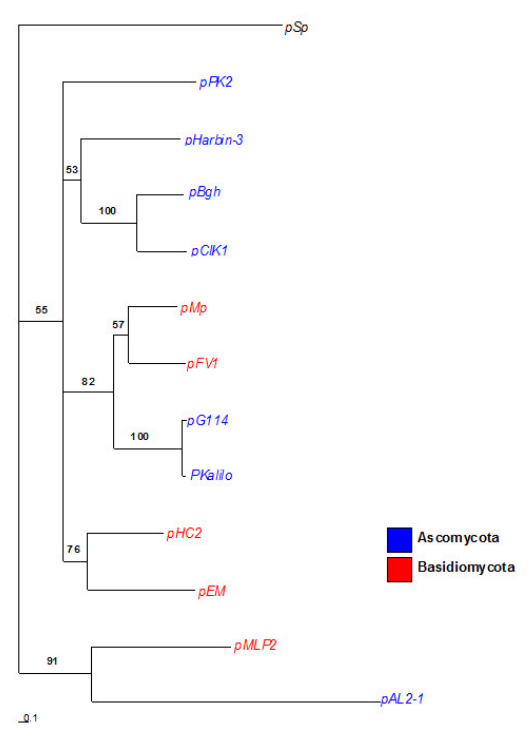

Figure 1. Phylogram (maximum likelihood) constructed using nucleotide sequence contigs of DNA and RNA polymerases from fungal linear mitochondrial plasmids. Numbers above branches are bootstrap values.

The Bayesian majority consensus tree built using amino acid sequences did not form a monophyletic clade of viral DPOs, but rather showed them to be interspersed among fungal DPOs (Figure 2). Conversely, Bayesian inference supported two homogeneous clades when using RPO protein sequences, one for fungal polymerases $(100 \%)$ and another for those of viruses (74\%; Figure 3).

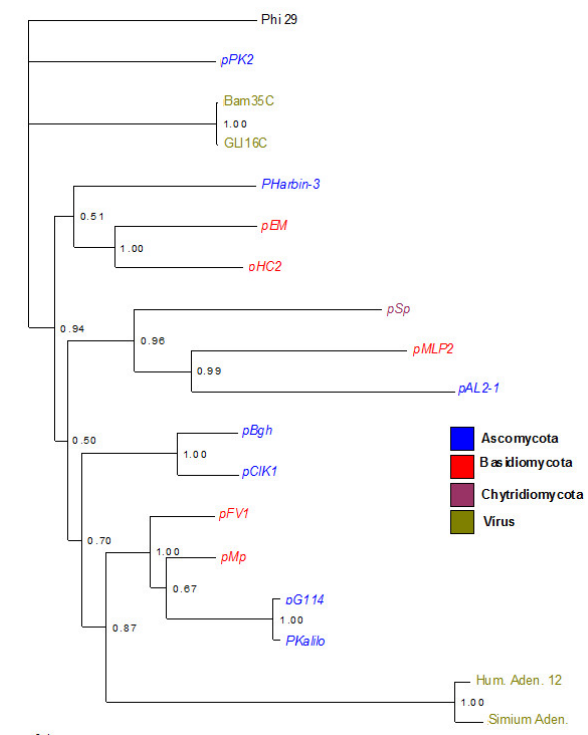

$-0.1$

Figure 2. Majority-rule consensus phylogram (Bayesian analysis) constructed using fungal and viral DNA polymerase amino acid sequences. 


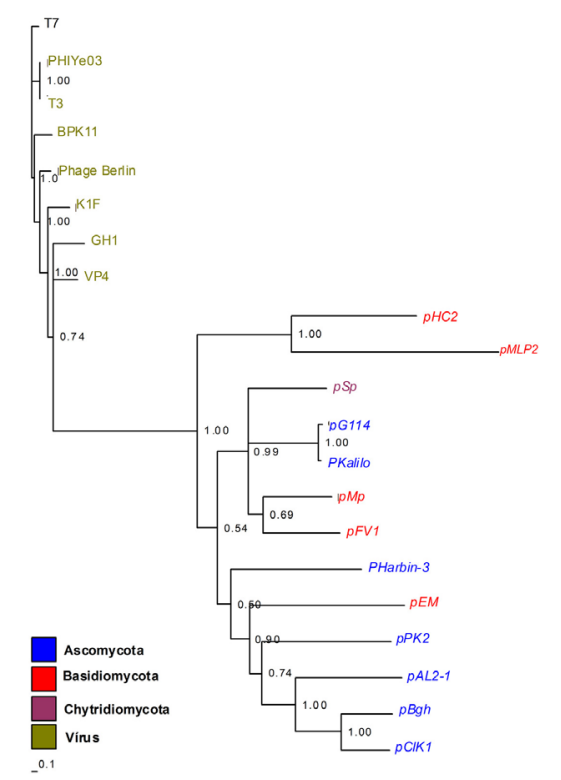

Figure 3. Majority-rule consensus phylogram (Bayesian analysis) constructed using fungal and viral RNA polymerase amino acid sequences.

\section{DISCUSSION}

According to Griffiths (1995), most fungal plasmids are linear and employ a mechanism similar to the protein-primed replication associated with adenoviruses and phage phi29. This process utilizes a terminal protein covalently linked to the 5'-end of these molecules. According to Sakaguchi (1990), adenovirus and phage phi29 genomes exhibit DNA plasmid-like features, with TIRs and terminal proteins covalently linked to their 5'-ends. All 13 plasmids examined in our study, with the exception of that from S. punctatus, have very similar, linear, structures, containing TIRs and DNA and RNA polymerases encoded in opposite orientations. Comparative sequence analysis revealed a probable ancestral relationship between the polymerases encoded by plasmids and those found in viruses. This suggests that the process by which these plasmids replicate is the same as that described by Griffiths (1995) for viral polymerases, thus corroborating the findings of Sakaguchi (1990).

There are several approaches to ascertaining the origin and distribution of plasmids in fungal mitochondria. According to Fukuhara (1995), it is highly likely that these structures derived from adenoviruses and phages within the bacterial ancestors of present-day mitochondria. This hypothesis is supported by the comparative sequence analysis carried out in this study, since fungal plasmid DPO and RPO sequences were found to be similar to those from adenoviruses and retroviruses, respectively. However, as described by Fukuhara (1995), such data do not address any hypothesis of how these structures came to be present in the cytoplasm of fungi.

Rosewich and Kistler (2000) reported many examples of lateral gene transfer (LGT) in fungi, in which mobile genetic elements, such as plasmids, transposons, and introns must be involved. Phylogenies created from mitochondrial plasmid DPO and RPO sequences, whether analyzed in combination or separately, revealed that Ascomycota and Basidiomycota polymerases do not form monophyletic groups, and therefore do not have a common origin. In addition, it is interesting to note 
that even DPOs and RPOs of species within the same genus ( $N$. crassa and N. intermedia) are not monophyletic, as inferred from phylogenetic analyses at both gene and protein levels.

A recent molecular phylogenetics study conducted by James et al. (2006), based on data from six nuclear genes in 199 species of fungi, strongly suggests that Ascomycota and Basidiomycota are monophyletic, while Chytridiomycota is clearly polyphyletic. Thus, the DNA and RNA polymerases of the 13 fungal mitochondrial plasmids examined here may be expected to reflect the monophyletic grouping of their Ascomycota and Basidiomycota host species. However, phylogenies based on both protein and DNA sequence data did not support this assumption.

The DPO phylogeny, including 13 fungal plasmids and their homologous viral polymerases, corroborated the supposed relationship between fungal DNA plasmids and adenovirus and bacteriophage sequences, as previously described. A monophyletic fungal group with high statistical support was retrieved only in the phylogeny of fungal and viral RPOs. When DPOs were analyzed, human adenovirus type 12 and simian adenovirus A were grouped within a strongly supported clade in the majority consensus tree, together with both ascomycete and basidiomycete sequences.

The DPO phylogeny using fungal mitochondrial plasmid and viral sequences was similar to that reported by Rosewich and Kistler (2000). These authors performed a phylogenetic analysis of DPO amino acid sequences, including 11 linear plasmids from fungi and plants, four bacteriophages, and eight viruses, finding that groups of plasmids with related hosts are not necessarily closely related themselves.

A likely explanation for the occurrence of groups not reflecting the monophyletic nature of their host species in the analysis of fungal and viral polymerases is that these enzymes may have originated during a pre-endosymbiotic period, during which the bacteria that subsequently became mitochondria hosted viruses and phages. This hypothesis has been put forward by Griffiths (1995) but it must be considered carefully. The lack of correspondence between the DPO and RPO groups and their monophyletic hosts may also be due to a post-endosymbiotic insertion event carried out by mycoviruses capable of transferring these polymerases into mitochondrial genomes. This assertion is based on observations described by Rosewich and Kistler (2000), in which the sequence of a viral RPO in the mitochondria of the ascomycete Ophiostoma novo-ulmi demonstrated greater similarity to that of the basidiomycete Rhizoctonia solani than to that of Cryphonectria parasitica, another ascomycete.

All phylogenetic trees generated using DNA and RNA polymerases from the 13 fungal plasmids, as well as those including both fungi and viruses, had very similar topologies. In the majority of consensus trees from both combined (contigs) and separate analyses, M. perniciosa plasmid polymerases were most closely related to those of Flammulina velutipes. Interestingly, in most analyses, the clade formed by these two basidiomycetes was more closely related to that including polymerases from the ascomycetes Gelasinospora sp. and N. intermedia than any other group.

According to van der Gaag et al. (1998), the transfer of mitochondrial plasmids between fungal species can occur via mitosis or meiosis. Indeed, the transfer of mitochondrial plasmid SP12 from Ascobolus immersus to Podospora pauciseta has been observed using co-culture experiments (Hermanns and Osiewacz, 1992). Thus, the close phylogenetic relationship observed in virtually all of our analyses between the clade formed by $M$. perniciosa and $F$. velutipes and that consisting of Gelasinospora sp. and N. intermedia may be the result of gene transfer at the genus or species level. This must be considered with caution and further studies of this more inclusive clade (M. perniciosa, F. velutipes, Gelasinospora sp., and N. intermedia) are required. An alternative explanation for this close relationship is the aforementioned mycovirus hypothesis, involving separate infections of fungal mitochondria by the same or very similar viruses. Furthermore, 
mycovirus sequences were consistently retrieved during the comparative sequence analysis when selecting the viral polymerases to be used in the construction of phylogenies.

The analysis based only on fungal plasmid and viral RPO amino acid sequences suggests a single origin for these enzymes in fungi. In addition, the clades retained in all trees, such as those formed by M. perniciosa and F. velutipes plasmid RPOs, indicate that the insertion of these sequences into fungal mitochondrial genomes occurred at short intervals, while the substitution rate in this organelle was equal for all species used in this study.

According to Formighieri et al. (2008), the disruptive integration of a plasmid into the mitochondrial genome of $M$. perniciosa was a recent event, and may have been associated with the development of certain biotypes. This process may have occurred through the crossing of distantly related lineages by the mitotic or meiotic mechanisms previously mentioned, or by mycoviruses. This was observed in all the trees that we generated, since the clade formed by $M$. perniciosa and F. velutipes was more closely related to that formed by Gelasinospora sp. and $N$. intermedia than to any other group. Furthermore, Bayesian analysis of amino acid sequences from fungal and viral DPOs suggested that the clade formed by $M$. perniciosa and $F$. velutipes appears to be closely related to viral polymerases. Therefore, in the RPO phylogeny including viral sequences, the formation of a monophyletic fungal clade suggests that plasmids encoding RPOs may have been inserted during events separate to those in which DPOs were introduced, both in M. perniciosa and other fungi.

Taking into account the assumptions made and results obtained from analyses of polymerase sequences within the fungal clade, and between fungi and viruses, we suggest that a lateral transfer of genes encoding DNA and RNA polymerases has occurred from the M. perniciosa plasmid to those of other fungal mitochondria, supporting the hypothesis that these enzymes may have viral ancestors. It is unclear whether these sequences date from a pre- or post-endosymbiotic period and this issue merits further investigation.

\section{CONCLUSIONS}

All phylogenetic analyses (parsimony, distance, maximum likelihood, and Bayesian) presented similar tree topologies, using both nucleotide and amino acid sequences in combined (contigs) or separate analyses, and exclusively fungal or fungal and viral datasets. While fungal DPOs did not group together, RPOs from fungal species formed a monophyletic group when compared to those derived from viruses. Furthermore, our study indicates that fungal DPOs and RPOs were probably inserted in distinct LGT events into the mitochondrial genomes of the organisms studied here.

\section{Conflicts of interest}

The authors declare no conflict of interest.

\section{ACKNOWLEDGMENTS}

We would like to acknowledge financial support from FAPESB (Fundação de Amparo à Pesquisa do Estado da Bahia) as well as the State University of Feira de Santana (UEFS) and its Graduate Program in Biotechnology (PPGBiotec, www.uefs.br/ppgbiotec). 


\section{REFERENCES}

Aime MC and Phillips-Mora W (2005). The causal agents of witches' broom and frosty pod rot of cacao (chocolate, Theobroma cacao) form a new lineage of Marasmiaceae. Mycologia 97: 1012-1022.

Altschul SF, Madden TL, Schäffer AA, Zhang J, et al. (1997). Gapped BLAST and PSI-BLAST: a new generation of protein database search programs. Nucleic Acids Res. 25: 3389-3402.

Bai X, Debaud JC, Schründer J, Meinhardt F (1997). The ectomycorrhizal basidiomycete Hebeloma circinans harbors a linear plasmid encoding a DNA- and RNA polymerase. J. Gen. Appl. Microbiol. 43: 273-279.

Baidyaroy D, Glynn JM and Bertrand H (2000). Dynamics of asexual transmission of a mitochondrial plasmid in Cryphonectria parasitica. Curr. Genet. 37: 257-267.

Blaisonneau J, Nosek J and Fukuhara H (1999). Linear DNA plasmid pPK2 of Pichia kluyveri: distinction between cytoplasmic and mitochondrial linear plasmids in yeasts. Yeast. 15: 781-91.

Bok JW, He C and Griffiths A (1999). Transfer of Neurospora kalilo plasmids among species and strains by introgression. Curr. Genet. 36: 275-281.

Cahan P and Kennel JC (2005). Identification and distribution of sequences having similarity to mitochondrial plasmids in mitochondrial genomes of filamentous fungi. Mol. Genet. Genomics 273: 462-473.

Chan BS, Court DA, Vierula PJ and Bertrand H (1991). The kalilo linear senescence-inducing plasmid of Neurospora is an invertron and encodes DNA and RNA polymerases. Curr. Genet. 20: 225-237.

Davison AJ, Akter P, Cunningham C, Dolan A, et al. (2003). Homology between thehuman cytomegalovirus RL11 gene family and human adenovirus E3 genes. J. Gen. Virol. 84: 657-63.

Dietz A, Weisser HJ, Kössel H, Hausmann R (1990). The gene for Klebsiella bacteriophage K11 RNA polymerase: sequence and comparison with the homologous genes of phages T7, T3, and SP6. Mol. Gen. Genet. 221: 283-6.

Dunn JJ, Studier FW and M. Gottesman (1983). Complete nucleotide sequence of bacteriophage T7 DNA and the locations of T7 genetic elements. J. Mol. Biol. 166: 477-535.

Forget L, Ustinova J, Wang Z, Huss VA, et al. (2002). Hyaloraphidium curvatum: a linear mitochondrial genome, tRNA editing, and an evolutionary link to lower fungi. Mol. Biol. Evol. 19: 310-9.

Formighieri EF, Tiburcio RA, Armas ED, Medrano FJ, et al. (2008). The mitochondrial genome of the phytopathogenic basidiomycete Moniliophthora perniciosa is $109 \mathrm{~kb}$ in size and contains a stable integrated plasmid. Mycol. Res. 112: 1136-1152

Fukuhara $\mathrm{H}$ (1995). Linear DNA plasmids of yeasts. FEMS Microbiol. Lett. 131: 1-9.

Giese H, Lyngkjaer MF, Stummann BM, Grell MN, et al. (2003). Analysis of the structure and inheritance of a linear plasmid from the obligate biotrophic fungus Blumeria graminis f. sp. hordei. Mol. Genet. Genomics 269: 699-705.

Griffith GW and Hedger JN (1994). The breeding biology of biotypes of the witches' broom pathogen of cocoa, Crinipellis perniciosa. Heredity 72: 278-289.

Griffith GW, Nicholson J, Nenninger A, Birch RN, et al. (2003). Witches' brooms and frosty pods: two major pathogens of cacao. New Zeal. J. Bot. 41: 423-435.

Griffiths AJ (1995). Natural plasmids of filamentous fungi. Microbiol. Rev. 59: 673-685.

Hall TA (1999). BioEdit: a user-friendly biological sequence alignment editor and analysis program for Windows 95/98/NT. Nucleic Acids Symp. Ser. 41: 95-98.

Henikoff S and Henikoff JG (1992). Amino acid substitution matrices from protein blocks. Proc. Natl. Acad. Sci. U S A 89: 10915-10919.

Hermanns $\mathrm{J}$ and Osiewacz HD (1992). The linear mitochondrial plasmid pAL2-1 of a long-lived Podospora anserina mutant is an invertron encoding a DNA and RNA polymerase. Curr. Genet. 22: 491-500.

James TY, Kauff F, Schoch CL, Matheny PB, et al. (2006). Reconstructing the early evolution of Fungi using a six-gene phylogeny. Nature 443: 818-822.

Kamtekar S, Berman AJ, Wang J, Lázaro JM, et al. (2004). Insights into strand displacement and processivity from the crystal structure of the protein-primed DNA polymerase of bacteriophage phi29. Mol. Cell. 16: 609-18. Erratum in: Mol. Cell. 16:1035-6.

Kempken F (1994). Unique features of a linear plasmid of Ascobolous immerses: Implications for plasmid evolution in fungi. Curr. Top. Mol. Genet. 2: 207-218.

Kempken F, Hermanns J and Osiewacz HD (1992). Evolution of linear plasmids. J. Mol. Evol. 35: 502-513.

Kim EK, Jeong JH, Youn HS, Koo YB, et al. (2000). The terminal protein of a linear mitochondrial plasmid is encoded in the $\mathrm{N}$-terminus of the DNA polymerase gene in white-rot fungus Pleurotus ostreatus. Curr. Genet. 38: 283-90.

Kovács GM, Davison AJ, Zakhartchouk AN, Harrach B (2004). Analysis of the first complete genome sequence of an Old World monkey adenovirus reveals a lineage distinct from the six human adenovirus species. J. Gen. Virol. 85: 2799-807. 
Kovalyova IV, Kropinski AM (2003). The complete genomic sequence of lytic bacteriophage gh-1 infecting Pseudomonas putida-evidence for close relationship to the T7 group. Virology. 311: 305-15.

MARCOU Marcou D (1961). Notion de llongéCvitéP et nature cytoplasmique du déeterminant de la séenescence chez quelques champignons. Ann. Sci. Nat. Bot. 12: 653-763.

Meinhardt F, Kempken F, Kämper J and Esser K (1990). Linear plasmids among eukaryotes: fundamentals and application. Curr. Genet. 17: 89-95.

Mondego JM, Carazzolle MF, Costa GG, Formighieri EF, et al. (2008). A genome survey of Moniliophthora perniciosa gives new insights into Witches' Broom Disease of cacao. BMC Genomics 9: 548.

Nakai R, Sen K, Kurosawa S, Shibai H (2000). Basidiomycetous fungus Flammulina velutipes harbors two linear mitochondrial plasmids encoding DNA and RNA polymerases. FEMS Microbiol. Lett. 190: 99-102.

Oeser B and Tudzynski P (1989). The linear mitochondrial plasmid pCIK1 of the phytopathogenic fungus Claviceps purpurea may code for a DNA polymerase and an RNA polymerase. Mol. Gen. Genet. 217: 132-40.

Osawa S, Jukes TH, Watanabe K and Muto A (1992). Recent evidence for evolution of the genetic code. Microbiol. Rev. 56: 229-264.

Page RD (1996). TreeView: an application to display phylogenetic trees on personal computers. Comput. Appl. Biosci. 12: 357-358.

Pajunen MI, Elizondo MR, Skurnik M, Kieleczawa J, et al. (2002). Complete nucleotide sequence and likely recombinatorial origin of bacteriophage T3. J. Mol. Biol. 319: 1115-32.

Pajunen MI, Kiljunen SJ, Söderholm ME, Skurnik M (2001). Complete genomic sequence of the lytic bacteriophage phiYeO3-12 of Yersinia enterocolitica serotype O:3. J. Bacteriol. 183: 1928-37.

Posada D and Crandall KA (1998). MODELTEST: testing the model of DNA substitution. Bioinformatics 14: 817-818.

Purdy LH and Schmidt RA (1996). STATUS OF CACAO WITCHES' BROOM: biology, epidemiology, and management. Annu. Rev. Phytopathol. 34: 573-594.

Ravantti JJ, Gaidelyte A, Bamford DH, Bamford JK (2003). Comparative analysis of bacterial viruses Bam35, infecting a grampositive host, and PRD1, infecting gram-negative hosts, demonstrates a viral lineage. Virology. 313: 401-14.

Robison MM and Horgen PA (1996). Plasmid RNA polymerase-like mitochondrial sequences in Agaricus bitorquis. Curr. Genet. 29: 370-6.

Ronquist F and Huelsenbeck JP (2003). MrBayes 3: Bayesian phylogenetic inference under mixed models. Bioinformatics 19: $1572-1574$

Rosewich UL and Kistler HC (2000). Role of horizontal gene transfer in the evolution of fungi. Annu. Rev. Phytopathol. 38: 325-363.

Sakaguchi K (1990). Invertrons, a class of structurally and functionally related genetic elements that includes linear DNA plasmids, transposable elements, and genomes of adeno-type viruses. Microbiol. Rev. 54: 66-74.

Scholl D and Merril C (2005). The Genome of Bacteriophage K1F, a T7-Like Phage That Has Acquired the Ability To Replicate on K1 Strains of Escherichia coli . J. Bacteriol. 187: 8499-8503.

Suyama M, Torrents D and Bork P (2006). PAL2NAL: robust conversion of protein sequence alignments into the corresponding codon alignments. Nucleic Acids Res. 34: W609-W612.

Swofford DL (2002). PAUP*: Phylogenetic Analysis Using Parsimony (*and Other Methods), Version 4. Sinauer Associates, Sunderland, Massachusetts.

van der Gaag M, Debets AJ, Osiewacz HD and Hoekstra RF (1998). The dynamics of pAL2-1 homologous linear plasmids in Podospora anserina. Mol. Gen. Genet. 258: 521-529.

Verheust C, Fornelos N and Mahillon J (2005). GIL16, a New Gram-Positive Tectiviral Phage Related to the Bacillus thuringiensis GIL01 and the Bacillus cereus pBClin15 Elements. J. Bacteriol. 187: 1966-1973.

Xu Y, Yang S, Turitsa I and Griffiths A (1999). Divergence of a linear and a circular plasmid in disjunct natural isolates of the fungus Neurospora. Plasmid 42: 115-125.

Yang Z, Nielsen R and Hasegawa M (1998). Models of amino acid substitution and applications to mitochondrial protein evolution. Mol. Biol. Evol. 15: 1600-1611.

Yuewang W, Yang X and Griffiths AJ (1996). Structure of a Gelasinospora linear plasmid closely related to the kalilo plasmid of Neurospora intermedia. Curr Genet. 29: 150-8. 